International Journal of Modern Physics A

(C) World Scientific Publishing Company

\title{
General thoughts to the Kaon pair production in the threshold region
}

\author{
W. Oelert ${ }^{\%, *}$, H.-H. Adam\#, A. Budzanowski ${ }^{\$}$, E. Czerwiński $^{\star}$, R. Czyżykiewicz ${ }^{\star}$, D. Gil ${ }^{\star}$, \\ D. Grzonka ${ }^{\%}$, M. Janusz ${ }^{\star}$, L. Jarczyk ${ }^{\star}$, B. Kamys ${ }^{\star}$, A. Khoukaz ${ }^{\#}$, P. Klaja ${ }^{\star}$, P. Moskal ${ }^{\star}$, \\ C. Piskor-Ignatowicz ${ }^{\star}$, J. Przerwa ${ }^{\star}$, T. Rożek ${ }^{+}$, R. Santo\#, T. Sefzick\%, M. Siemaszko ${ }^{+}$, \\ J. Smyrski ${ }^{\star}$ A. Täschner\#, P. Winter ${ }^{\star}$, M. Wolke $\%$, P. Wüstner ${ }^{\otimes}$, W. Zipper ${ }^{+}$, \\ \% IKP, Forschungszentrum Jülich, Jülich, Germany \\ \# IKP, Westfälische Wilhelms-Universität, Münster, Germany \\ \$Institute of Nuclear Physics, Cracow, Poland \\ *Institute of Physics, Jagellonian University, Cracow, Poland \\ ${ }^{+}$Institute of Physics, University of Silesia, Katowice, Poland \\ $\times$ Department of Physics, University of Illinois at Urbana-Champaign, Urbana, IL 61801 USA \\ $\otimes Z E L$, Forschungszentrum Jülich, Jülich, Germany
}

\begin{abstract}
Simple-minded thoughts about the cross sections for the reactions $p p \rightarrow p p K^{+} K^{-}$and $p p \rightarrow p p K^{0} K^{0}$ are presented, which certainly do not account for the complex coupled channel problem but rather provide some ideas into the mutual reaction dynamics.
\end{abstract}

Keywords: Hyperon - Cross Sections

PACS. 13.60.Hb; 13.60.Le; 13.75.-n; 25.40.Ve

\section{Introduction}

Total cross sections $\frac{1|2| 3 \mid 4}{4}$ of the reaction $p p \rightarrow p p K^{+} K^{-}$at excess energies below $Q=120 \mathrm{MeV}$ are given in Figure 1. At low excess energies the data points lie significantly above the various expectations indicated by the different lines as long as these predicted curves are all normalized to the DISTO point 4 at $Q=114 \mathrm{MeV}$. The pure phase space (dashed line) differs by two orders of magnitude at $Q=$ $10 \mathrm{MeV}$ and a factor of five to ten at $Q=28 \mathrm{MeV}$. In comparison to that, the inclusion of the $p p$-FSI (dashed-dotted line) by folding its parameterization known from the three body final state with the four body phase space is already closer to the experimental results but does not fully account for the difference. The solid line representing the calculation within a one-boson exchange model 5 reveal a similar discrepancy as the $p p$-FSI parameterization. This model includes an energy dependent scattering amplitude derived from the fit of the total cross sections in $K^{ \pm} p \rightarrow K^{ \pm} p[6$ while the $p p$-FSI was not included, yet. Up to now, there is no full calculation available but the new data demands for further theoretical efforts in order to give a complete picture of the $K^{+} K^{-}$production.

$\overline{{ }^{*} \text { E-mail address: w.oelert@fz-juelich.de }}$ 


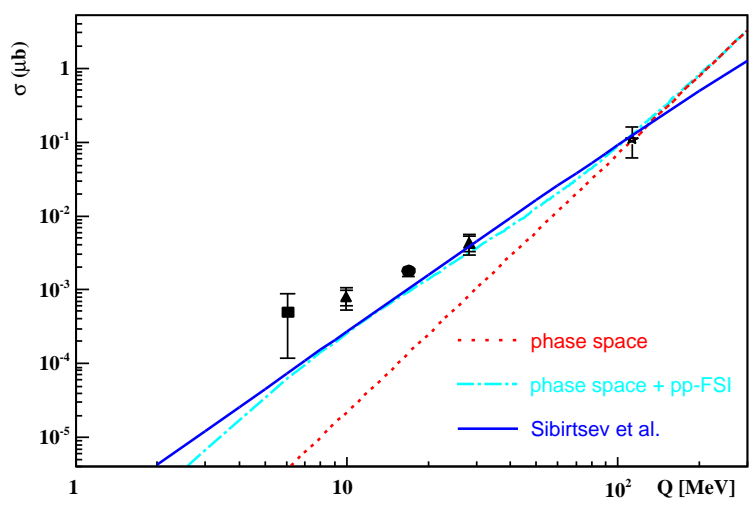

Fig. 1. Total cross section as a function of the excess energy $Q$ for the reaction $p p \rightarrow p p K^{+} K^{-}[1 / 2|3| 4$.

\section{The model}

An important aspect might be the mass splitting between the neutral $K^{0} \bar{K}^{0}$ and charged $K^{+} K^{-}$kaons being in the order of $8 \mathrm{MeV}$. Based on the theoretical observation that the opening neutral kaon production channel shows a substantial influence on the $\pi \pi \rightarrow K^{+} K^{-}$transition (c.f. Figure 2. in reference 7 ), we tried a simple-minded Ansatz for the energy dependence of the excitation function for the $p p \rightarrow p p K^{+} K^{-}$reaction assuming that with the opening of the neutral kaon channel (at $8 \mathrm{MeV}$ excess energy) some yield is taken out of the charged kaon channel. As long as the total energy is large enough to produce the charged kaon pair but is below the neutral kaon channel all strength for the associated strangeness production is devoted to the $K^{+} K^{-}$creation. At $8 \mathrm{MeV}$ excitation energy the charged channel faces the competition of the neutral one.

\section{Cross section extraction}

For estimating such a coupled channel effect, we postulated the two simple assumptions. First, we assume that the excitation function for the neutral $p p \rightarrow p p K^{0} \bar{K}^{0}$ channel follows exactly the dashed-dotted line in figure 1 for the charged kaon production case, but shifted by $8 \mathrm{MeV}$. If $f(Q)$ describes the excitation function of the dashed-dotted line, we simply assume that the energy dependence for the neutral channel is given by $g(Q)=f(Q-8 \mathrm{MeV})$ where $Q$ refers here to the $K^{+} K^{-}$system. Second, the opening channel is incorporated by the idea that the modified description $\bar{f}(Q)$ of the $p p \rightarrow p p K^{+} K^{-}$channel is given by a subtraction of the neutral channel via $\bar{f}(Q):=c \cdot[f(Q)-g(Q)]$ with an arbitrary normalization $c$. This is certainly an extreme scenario since the influence of the $K^{0} \bar{K}^{0}$ channel is assumed to be an uncorrelated sum of the $K^{+} K^{-}$and $K^{0} \bar{K}^{0}$ cross sections; and, since the inverse transition $K^{0} \bar{K}^{0} \rightarrow K^{+} K^{-}$is not considered to take place. The resulting 


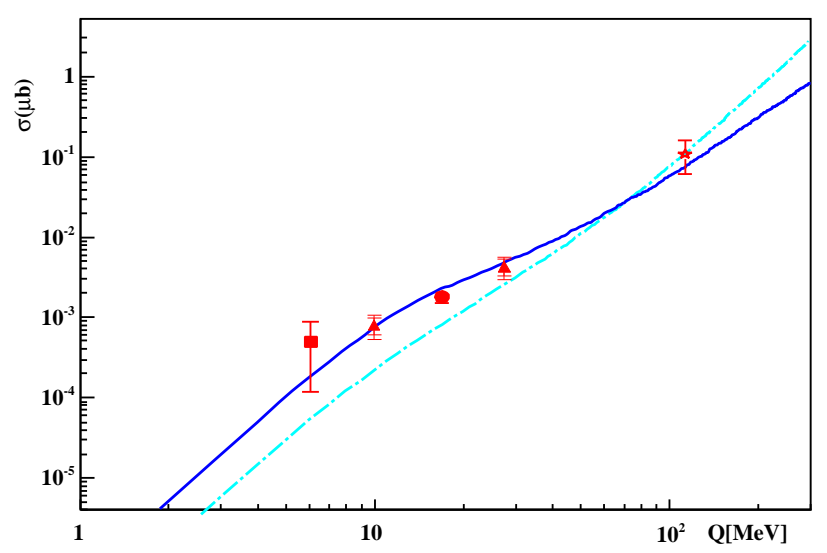

Fig. 2. Experimental data together with a simplified Ansatz for the incorporation the $K^{0} \bar{K}^{0}$ channel (solid line).

energy dependence $\bar{f}(Q)$ as shown in Figure 2 just happens to pass through the experimental data.

This good agreement should be taken with caution since a full coupled channel calculation has to be performed to determine quantitatively the effect of the $K^{0} \bar{K}^{0}$ channel, which is expected to be rather small 8 and can not account for the full enhancement seen in the data. Furthermore other effects are not considered which should certainly be taken fully into account such as final state interactions between the subsystems $p-K^{+}, p-K^{-}$and $K^{+}-K^{-}$as well as the influence of intermediate resonances.

We acknowledge the support of the European Community-Research Infrastructure Activity under the FP6 "Structuring the European Research Area" programme (HadronPhysics, contract number RII3-CT-2004-506078), of the FFE grants (41266606 and 41266654) from the Research Centre Jülich, of the DAAD Exchange Programme (PPP-Polen), of the Polish State Committe for Scientific Research (grant No. PB1060/P03/2004/26), and of the RII3/CT/2004/506078 - Hadron PhysicsActivity -N4:EtaMesonNet.

\section{References}

1. P. Winter et al., Phys. Lett. B 63523 (2006) arXiv:hep-ex/0602030.

2. C. Quentmeier et al., Phys. Lett. B 515276 (2001) arXiv:hep-ex/0103001.

3. M. Wolke, Dissertation, Münster, 1997, IKP JÜL. 3532

4. F. Balestra et al., Phys. Rev. C 63024004 (2001) arXiv:hep-ex/0011009.

5. A. Sibirtsev, et al., Z. Phys. A 358101 (1997) arXiv:hep-ex/9612040].

6. A. Baldini et al., Landolt-Börnstein, Vol I/12, New Series, Springer, Berlin, 1988

7. O. Krehl, et al., Phys. Lett. B 39023 (1997) arXiv:hep-ex/9609013.

8. J. Haidenbauer, private communication 